PROCEEDINGS OF THE

AMERICAN MATHEMATICAL SOCIETY

Volume 49, Number 1, May 1975

\title{
LINEAR CONNECTIONS AND ALMOST COMPLEX STRUCTURES
}

\author{
JEAN-MARC TERRIER ${ }^{1}$
}

ABSTRACT. An almost complex structure is defined on $P$, the principal bundle of linear frames over an arbitrary even-dimensional smooth manifold $M$ with a given linear connection. Complexifying connections are those which induce a complex structure on $P$. For twodimensional manifolds, every linear connection is of this kind.

In the special case where $M$ itself is an almost complex manifold, a relationship between the two almost complex structures is found and provides a very simple proof of the fact that the existence of an almost complex connection without torsion implies the integrability of the given almost complex structure. As a second application, we give a geometrical interpretation of an identity between the torsion of an almost complex structure on $M$ and the torsion of an almost complex connection over $M$.

1. Introduction. In this paper we associate to each linear connection over an arbitrary even-dimensional smooth manifold $M$ an almost complex structure $\hat{J}$ on $P$, the principal bundle of linear frames over $M$. This almost complex structure actually depends on three objects: a linear connection, a complex structure on $\mathbf{R}^{m}(m=2 n=\operatorname{dim} M)$, and a complex structure on the Lie algebra of the general linear group GL $(m ; \mathbf{R})$. Theorem 2.2 gives the explicit relationship. Theorem 3.2 expresses the torsion of the almost complex structure $\hat{J}$ in terms of the curvature and the torsion of the connection via a construction-the mixed torsion map-which turns out to be, by Theorem 3.3, a singular endomorphism of 2 -forms on $P$ with values in a complex vector space. This suggests calling complexifying connections those connections which induce a complex structure on $P$. For instance, Theorem 3.4 shows that any linear connection on a two-dimensional manifold is a complexifying connection.

Received by the editors July 25, 1973 and, in revised form, April 1, 1974. AMS (MOS) subject classifications (1970). Primary 32C 10, 53C15; Secondary 53B35, 53C05, 53C10, 53C55.

Key words and phrases. Almost complex structures, complex manifolds, linear connections, complexifying connections, almost complex connections.

1 Research supported by the Canadian Mathematical Congress through a Summer Institute grant and by a grant of the Gouvernement du Québec. 
For the rest of the paper, we shall assume that $M$ admits an almost complex structure $J_{M}$. Theorem 4.1 gives a way to compare $J_{M}$ with $\hat{J}$. In the case of an almost complex connection, formula (3.1) first provides a direct proof of the converse of Proposition 1.2 in [3] or of the necessary part of Corollary 3.5 in [2]. Secondly, it gives a geometric interpretation of the fact that the torsion $T$ of an almost complex connection on an almost complex manifold $\left(M, J_{M}\right)$ satisfies the identity [2, Proposition 3.6]

$$
\tau_{M}(X, Y)=T\left(J_{M} X, J_{M} Y\right)-T(X, Y)-J_{M} T\left(J_{M} X, Y\right)-J_{M} T\left(X, J_{M} Y\right)
$$

The reader is referred to [1, Chapters I-IV] and [2, Chapter IX] for basic notions and notations.

2. Almost complex structures on the bundle of linear frames. Let us consider an even-dimensional smooth manifold, $\operatorname{dim} M=m=2 n$. Let $P$ be the principal bundle of linear frames over $M$ with projection $\pi$. Denote by $J$ (resp. $J_{0}$ ) the canonical complex structure of $\mathbf{R}^{m}$ [2, p. 115] (resp. $g\lfloor(m ; \mathbf{R})$, the Lie algebra of the general linear group $\mathrm{GL}(m ; \mathbf{R})$, identified with the Lie algebra of all $m \times m$ real matrices). $J$ is represented in the standard basis of $\mathbf{R}^{m}$ by the matrix

$$
\left(\begin{array}{cc}
0 & I_{n} \\
-I_{n} & 0
\end{array}\right)
$$

where $I_{n}$ is the identity $n \times n$ matrix; $J_{0}$ acts on $g\lfloor(m ; \mathbf{R})$ by left multiplication with the same matrix.

Suppose now we are given a linear connection $\Gamma$ in $P$ with $\omega$ as its 1 -form of connection. Using the fact that $\Gamma$ induces in each point $u \in P$ a direct sum decomposition of $T_{u}(P)$, the tangent space of $P$ at $u$, into a vertical and a horizontal part, we make the following definition.

Definition 2.1. Let us call $\hat{J}$ the $(1,1)$-tensor field on $P$ given by

$$
\hat{J} X=\lambda J_{0} \omega X+B J \theta X \quad \forall u \in P, \forall X \in T_{u}(P),
$$

where

(i) $\theta$ is the canonical $\mathbf{R}^{m}$-valued 1 -form on $P$,

(ii) $\lambda$ is the isomorphism between $g\lfloor(m ; \mathbf{R})$ and the fundamental vector fields on $P$,

(iii) $B(\xi)$ is the standard horizontal vector field on $P$ corresponding to $\xi \in \mathbf{R}^{m}$, whose value at $u$ is the unique horizontal vector such that 
$\pi\left(B(\xi)_{u}\right)=u(\xi)$, where $u$ is interpreted as a linear isomorphism: $\mathbf{R}^{m} \rightarrow$ $T_{\pi(u)}(M)$.

We have the following theorem.

Theorem 2.2. If $M$ is an even-dimensional smooth manifold with linear connection $\Gamma, J$ (resp. $J_{0}$ ) the canonical complex structure on $\mathbf{R}^{m}$ (resp. on $\mathrm{gl}(m ; \mathbf{R}))$, then:

(1) the $(1,1)$-tensor field $\cdot \hat{\mathcal{J}}$ defined on $P$ by

$$
\hat{J}=\lambda J_{0} \omega+B J \theta
$$

is an almost complex structure on $P$.

(2) If $\omega^{\prime}=\omega+\eta$ is another connection 1-form with $\eta$ a tensorial form of type adj $\mathrm{GL}(m ; \mathbf{R})$, then the corresponding almost complex structure $\hat{J}^{\prime}$ on $P$ is given by

$$
\hat{J}^{\prime}=\hat{J}+\lambda\left(J_{0} \eta-\eta B J \theta\right) .
$$

(3) If $J_{S}$ (resp. $J_{Q}$ ) is a complex structure of $\mathbf{R}^{m}$ (resp. gL $(m ; \mathbf{R})$ ) corresponding to the nontrivial left coset $[\mathrm{S}]$ in $\mathrm{GL}(m ; \mathbf{R}) / \mathrm{GL}(n ; \mathbf{C})$ (resp. $[Q]$ in $\left.\mathrm{GL}\left(\mathrm{m}^{2} ; \mathbf{R}\right) / \mathrm{GL}\left(2 n^{2} ; \mathbf{C}\right)\right)$, then the induced almost complex structure on $P$ is given by

$$
\hat{J}_{Q, S}=-\left(\lambda J_{Q} J_{0} \omega+B J_{S} J \theta\right) \hat{J} .
$$

Proof. (1) Because $\omega$ (resp. $\theta$ ) is a vertical (resp. horizontal) form and $\theta B=1$, we easily have $\hat{J}^{2}=-1$.

(2) A standard horizontal vector field $B^{\prime}$ in the new connection $\omega^{\prime}=$ $\omega+\eta$ is given by $B^{\prime}=B-\lambda \eta B$, because, for each $\xi \in \mathbf{R}^{m}, \eta B^{\prime}(\xi)=$ $-\omega B^{\prime}(\xi)$ and $\omega \lambda+B \theta=1$.

(3) By definition, $\hat{J}_{Q, s}=\lambda J_{Q} \omega+B J_{s} \theta$, where $J_{Q}=Q J_{0} Q^{-1}$ and $J_{s}=$ $S J S^{-1}$. The formula follows because $\omega \hat{J}=J_{0} \omega$ and $\theta \hat{J}=J \theta$.

From now on, we shall refer to the almost complex structure $\hat{j}$ defined above as the canonical almost complex structure corresponding to the condition $\omega$ or simply the $\omega$-canonical almost complex structure on $P$.

3. Complexifying connections. It is known that a necessary and sufficient condition for an almost complex structure $\hat{J}$ to be integrable is the vanishing of its torsion $\hat{r}$ defined by

$$
\tilde{\tau}(X, Y)=[X, Y]+\hat{J}[\hat{J} X, Y]+\hat{J}[X, \hat{J} Y]-[\hat{J} X, \hat{J} Y],
$$

where $X$ and $Y$ are vector fields on $P$.

Definition 3.1. For each $a \in \Lambda^{2}(P, V)$, a 2-form on $P$ with values in 
a vector space $V$ with a complex structure $J_{V}$, let $\tilde{\alpha} \in \Lambda^{2}(P, V)$ defined by

$$
-1 / 2 \tilde{\alpha}(X, Y)=\alpha(X, Y)+J_{V} \alpha(\hat{J} X, Y)+J_{V} \alpha(X, \hat{J} Y)-\alpha(\hat{J} X, \hat{J} Y)
$$

Theorem 3.2. If $\Gamma$ is a linear connection over $M$ with $\omega$ as its 1-form of connection, then the torsion $\hat{\tau}$ of the $\omega$-canonical almost complex structure $\hat{J}$ on $P$ is given by

$$
\hat{\tau}=\lambda \widetilde{\Omega}+B \widetilde{\Theta},
$$

where $\Omega$ (resp. $\Theta$ ) is the curvature form (resp. the torsion form of $\Gamma$ ).

Proof. Since each connection induces a parallelization of $P$ by means of the vector fields $B_{i}=B\left(e_{i}\right), 1 \leq i \leq m$, where $\left\{e_{1}, \cdots, e_{m}\right\}$ is the standard basis of $\mathbf{R}^{m}$, and the vector fields $X_{i}^{j}=\lambda E_{i}^{j}, 1 \leq i, j \leq m$, where $\left(E_{i}^{j}\right)_{1 \leq i, j \leq m}$ is the basis of $g l\left(m\right.$; R) defined by $\left(E_{i}^{j}\right)_{l}^{k}=\delta_{l}^{j} \delta_{i}^{k}$, it is sufficient to compute the torsion in the three following cases:

(a) $X$ and $Y$ are horizontal; more precisely $X=B_{1}$ and $Y=B_{2}$, where $B_{i}=B\left(\xi_{i}\right), \xi_{1}$ and $\xi_{2}$ linearly independent in $\mathbf{R}^{m}$.

(b) $X$ is horizontal; $X=B(\xi)$ for $\xi$ nonzero in $\mathbf{R}^{m}$ and $Y$ is vertical, say $Y=\lambda A$ where $A$ nonzero in $g\lfloor(m, \mathbf{R})$.

(c) $X$ and $Y$ are vertical; $X=\lambda A_{1}$ and $Y=\lambda A_{2}$ with $A_{1}$ and $A_{2}$ linearly independent in $g l(m ; \mathbf{R})$.

Using the structure equations $[1$, p. 120] for $\Omega$ and $\Theta$, we first get $\left[B_{1}, B_{2}\right]=-2 \lambda \Omega\left(B_{1}, B_{2}\right)-2 B \Theta\left(B_{1}, B_{2}\right)$. Secondly by $[1, \mathrm{p} .119]$ we have $[B(\xi), \lambda A]=-B(A \xi)$, and finally, $\left[\lambda A_{1}, \lambda A_{2}\right]=\lambda\left[A_{1}, A_{2}\right]$.

Observing that $\hat{J} B(\xi)=B(J \xi)$ since $\theta B=1$, and $\hat{J} \lambda A=\lambda J_{0} A$ since $\omega \lambda=1$, the theorem follows by computation and use of Definition 2.1.

This result suggests calling a complexifying connection a connection whose curvature and torsion satisfy the conditions $\widetilde{\Omega}=0$ and $\widetilde{\Theta}=0$. Then Theorem 3.2 can be stated in the following way:

Theorem 3.2'. The $\omega$-canonical almost complex structure on $P$ induced by a connection $\Gamma$ is integrable if and only if $\Gamma$ is a complexifying connection.

A flat linear connection on $M$, without torsion, gives a (rather trivial) example of such a connection, since $\Omega=0$ (resp. $\Theta=0$ ) implies $\tilde{\Omega}=0$ (resp. $\widetilde{\Theta}=0$ ). However, it is important to note that, in general, the form $\tilde{\alpha}$ can be zero without a being zero. More precisely we have the following theorem. 
Let us first call mixed torsion map the endomorphism $\mu$ of $\Lambda^{2}(P, V)$ defined by $\mu \alpha=-1 / 2 \tilde{\alpha}$. The name is suggested by the formal resemblance to Definition 3.1 of $\tilde{\tau}$, the structures $J_{V}$ and $\hat{J}$ being "mixed" together, and al so by formula (3.2).

Theorem 3.3. The mixed torsion map $\mu$ is singular.

Prool. The fact that $\mu$ is linear is trivial. To show that the kernel of $\mu$ is different from zero, let us first note that $\mu^{2}-4 \mu=0$ by an easy computation. On the other hand, $\mu \neq 4 I$ by the Lemma below (whose proof was kindly suggested to the author by Professor S. Takahashi). Suppose now that $\mu$ is nonsingular, then $(\mu-4) \alpha$ is in the kernel of $\mu$ for each $\alpha$, hence so is $\mu \alpha=$ $4 \alpha$, which gives a contradiction. Q.E.D.

Lemma. For the mixed torsion map $\mu$ we have $\mu \neq 4 I$.

Proof. Write $V=V_{0} \oplus J_{V} V_{0}$, viewing $V$ as a complex space and $V_{0}$ as the set of $a \in V$ such that $\bar{a}=\alpha$ (bar denotes complex conjugation). Accordingly we write $a=a^{+}+J_{V} a^{-}$for each $a \in V$. It is sufficient to show that $\mu=4 I$ and $\alpha^{-}=0$ implies $\alpha^{+}=0$. By definition of $\mu$, we have

$$
3 \alpha(X, Y)=J_{V} \alpha(\hat{J} X, Y)+J_{V} \alpha(X, \hat{J} Y)-\alpha(\hat{J} X, \hat{J} Y)
$$

Thus,

$$
3 \alpha^{+}(X, Y)=-\alpha^{+}(\hat{J} X, \hat{J} Y) \text { and } \alpha^{+}(X, Y)=\alpha^{+}(\hat{J} X, \hat{J} Y)
$$

Hence $4 \alpha^{+}(X, Y)=0$. Q.E.D.

For low-dimensional manifolds we have the following result.

Theorem 3.4. Each linear connection on a 2-dimensional manifold is a complexifying connection.

Proof. Take any nonzero element in $\mathbf{R}^{2}$ and call $B$ the standard horizontal vector field corresponding to it. By Theorem 3.2, it is sufficient to show that $\tilde{\alpha}(B, \hat{J} B)=0$, where $\alpha=\Omega$ (resp. $\Theta$ ), because these are horizontal 2-forms. By the skew symmetry of $\alpha$ and Definition 3.1 we have $-1 / 2 \tilde{a}(B, \hat{J} B)=\alpha(B, \hat{J} B)+J_{V} \alpha(\hat{J} B, \hat{J} B)-J_{V} \alpha(B, B)+\alpha(\hat{J} B, B)=0 . \quad$ Q.E.D.

Remark. Using the parallelization of $P$ given by a connection $\Gamma$, we can introduce a Riemannian metric on $P$ in a standard way, and it is easy to verify that with respect to this metric, $\Gamma$ induces an almost hermitian structure on $P$, which is hermitian in case $\Gamma$ is a complexifying connection. How- 
ever, such an (almost) hermitian structure is never (almost) Kählerian, as one can easily check.

4. Almost complex structures, up and down. From now on, we shall assume that $M$, itself, admits an almost complex structure $J_{M}$. It is known that the existence of an almost complex structure $J_{M}$ on $M$ is equivalent to the reduction of $L(M)$ to $C(M)$, the bundle of complex linear frames, the subbundle of $L(M)$ defined by $C(M)=\left\{u \in L(M) ; u J=J_{M} u\right\}$. (We view $u$ as in Definition 2.1(iii).) Let $\omega$ be any linear connection on $M$. If $X$ is a vector field on $X$ and $X^{*}$ its horizontal lift to $L(M)$, we can compare $\hat{J} X^{*}$ with $\left(J_{M} X\right)^{*}$ in the following theorem.

Theorem 4.1. For each vector field $X$ on $M$, we have $\hat{J} X^{*}=\left(J_{M} X\right)^{*}$ on $C(M)$.

Proof. For an arbitrary point $u$ in $L(M)$ we have by definition of $\hat{j}$, $\left(\hat{J} X^{*}\right)_{u}=\left(B J \theta\left(X_{u}^{*}\right)\right)_{u}=Y$, the unique horizontal vector at $u$ such that $\pi Y=$ $u J \theta\left(X_{u}^{*}\right)=u J u^{-1} X_{\pi(u)}$. On the other side $\left(J_{M} X\right)_{u}^{*}=Z$, the unique horizontal vector at $u$ such that $\pi Z=J_{M} X_{\pi(u)}$. Therefore

$$
Y=Z \Leftrightarrow u J u^{-1} X_{\pi(u)}=J_{M} X_{\pi(u)} \Leftrightarrow u J u^{-1}=J_{M} \Leftrightarrow u \in C(M) \text {. Q.E.D. }
$$

From now on we shall assume that $\omega$ is the 1-form of an almost complex connection, i.e. a linear connection arising from a connection on $C(M)$. This is known to be equivalent with the fact that $J_{M}$ is parallel with respect to this connection. Still denoting by $\hat{J}$ the corresponding almost complex structure on $L(M)$, we have the following relationship between its torsion $\hat{\tau}$ and ${ }^{\tau_{M}}$, the torsion of $J_{M}$.

Theorem 4.2. If $\left(M, J_{M}\right)$ is an almost complex manifold, and if $\omega$ is the 1-form of an almost complex connection on $M$, then

$$
B \widetilde{\Theta}\left(X^{*}, Y^{*}\right)=\left(\tau_{M}(X, Y)\right)^{*}
$$

on $C(M)$, where $X$ and $Y$ are vector fields on $M$.

Proof. According to (3.2) the torsion of $\hat{J}$ is given by $\hat{\tau}\left(X^{*}, Y^{*}\right)=$ $\lambda \tilde{\Omega}\left(X^{*}, Y^{*}\right)+B \tilde{\Theta}\left(X^{*}, Y^{*}\right)$. We are interested only in the second term of the right member, the first one being in the kernel of the differential of $\pi$. Formula (3.1) gives

$$
\hat{\tau}\left(X^{*}, Y^{*}\right)=\left[X^{*}, Y^{*}\right]+\hat{J}\left[\hat{J} X^{*}, Y^{*}\right]+\hat{J}\left[X^{*}, \hat{J} Y^{*}\right]-\left[\hat{J} X^{*}, \hat{J} Y^{*}\right]
$$

If we restrict ourselves to $C(M)$ then, by Theorem 2.2 and the very defini- 
tion of an almost complex connection, the horizontal part of the right member of this equation becomes

$$
[X, Y]^{*}+\left(J_{M}\left[J_{M} X, Y\right]\right)^{*}+\left(J_{M}\left[X, J_{M} Y\right]\right)^{*}-\left[J_{M} X, J_{M} Y\right]^{*},
$$

which is exactly the horizontal lift of the vector field $\tau_{M}(X, Y)$. Q.E.D.

This theorem has two interesting and simple applications. The first one is the converse (in the case of almost complex structures) of the general theorem [3], that if a $G$-structure on $M$ is integrable it admits a torsionfree connection. We have

Corollary 4.3. If an almost complex manifold $\left(M, J_{M}\right)$ admits a torsionfree almost complex connection, then $J_{M}$ is integrable.

Proof. $\Theta=0$ implies $\widetilde{\Theta}=0$ and, by Theorem 4.2, $\tau_{M}=0$.

The second application is a geometric interpretation of the identity (1.1) which is simply the projection down to $M$ of the torsion of $\hat{J}$ evaluated on horizontal lifts in $C(M)$. This is a consequence of the definition of the torsion $T$ of the connection as $T(X, Y)=u 2 \Theta\left(X^{*}, Y^{*}\right)$ and of $\widetilde{\Theta}$ as $-1 / 2 \tilde{\Theta}\left(X^{*}, Y^{*}\right)=\Theta\left(X^{*}, Y^{*}\right)+J \Theta\left(\hat{J} X^{*}, Y^{*}\right)+J \Theta\left(X^{*}, \hat{J} Y^{*}\right)-\Theta\left(\hat{J} X^{*}, \hat{J} Y^{*}\right)$.

\section{REFERENCES}

1. S. Kobayashi and K. Nomizu, Foundations of differential geometry. Vol. I, Interscience, New York, 1963. MR 27 \#2945.

2. - Foundations of differential geometry. Vol. II, Interscience Tracts in Pure and Appl. Math., no. 15, Interscience, New York, 1969. MR 38 \#6501.

3. S. Kobayashi, Transformation groups in differential geometry, Ergebnisse der Mathematik und ihrer Grenzgebiete, Band 70, Springer-Verlag; Berlin and New York, 1972.

DÉPARTEMENT DE MATHÉMATIQUES, UNIVERSITÉ DE MONTRÉAL, MONTRÉAL, QUÉBEC, CANADA 